\title{
When does the risk of acute coronary heart disease in ex-smokers fall to that in non-smokers? A retrospective study of patients admitted to hospital with a first episode of myocardial infarction or unstable angina
}

\author{
KILLIAN ROBINSON, RONAN M CONROY, RISTEARD MULCAHY \\ From the Cardiac Department and Department of Preventive Cardiology, St Vincent's Hospital and \\ University College, Dublin, Ireland
}

SUMMARY Nine hundred and seventy eight patients admitted with a first myocardial infarction or $\vec{c}$ episode of unstable angina were studied to determine for how long after they gave up smoking did the risk in ex-smokers continue to resemble those of current smokers. Logistic regression was used $\vec{\oplus}$ to calculate a score, based on a combination of age, cholesterol, and hypertension, that separated $\infty$ current smokers from lifetime non-smokers. When this function was applied to ex-smokers, only i those who had given up at least 15 years before the attack had a risk factor profile similar to that of $\sum^{\circ}$ non-smokers. Those who had given up less than five years before the ischaemic attack had a significantly higher level of other risk factors than current smokers; those who had stopped for between five and 15 years had levels similar to those of current smokers.

Ex-smokers are at higher risk of acute coronary disease for at least 15 years after stopping, but $\overrightarrow{\overrightarrow{\vec{O}}}$ some immediate reduction in risk is possible.

Cook et al reported that former smokers may be at a significantly greater risk of developing acute coronary heart disease for up to 20 years after giving up smoking than people who have never smoked. ${ }^{1}$ Earlier studies had suggested that the risk diminished more rapidly after stopping, ${ }^{2-8}$ with ex-smokers showing a significant ${ }^{2-5}$ or almost complete ${ }^{6-8}$ reduction in risk in as little as two years after giving up.

Most data on the effect of stopping smoking on subsequent risk have been drawn from prospective population studies, but the low incidence of acute episodes and the small numbers of ex-smokers may mean that all but the largest prospective studies lack the statistical power to detect modest differences in risk between ex-smokers and non-smokers (that is those who have never smoked).

Studies of patients experiencing a first acute episode of coronary heart disease can be used to clarify the role of individual risk factors. ${ }^{9}$ If we regard

Requests for reprints to Mr Ronan M Conroy, Cardiac Department, St Vincent's Hospital, Elm Park, Dublin 4, Ireland.

Accepted for publication 4 April 1989 this episode as marking a definable level of the progress of coronary heart disease, we would expect current smokers to have lower levels of other risk? factors-cholesterol, hypertension, and age-than patients who have never smoked. The risk factor "profile" that discriminated best between current and non-smokers could be applied to ex-smokers. If it differed from that found in non-smokers, the exsmokers must have had an acute episode of coronary $\frac{7}{0}$ heart disease despite a lower level of the risk factors of cholesterol, hypertension, and age. Such a result $N$ implies a continued risk derived from their previous: $N$ smoking.

We used multiple logistic regression analysis to estimate the length of time after the end of smoking for which ex-smokers are at a significantly greater

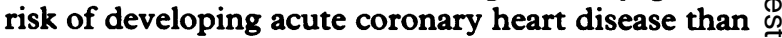
those who have never smoked.

\section{Patients and methods}

From January 1980 to April 1985 inclusive, all patients admitted to the coronary care unit of St 
Vincent's Hospital with a first episode of unstable angina or myocardial infarction were included in a computerised register.

Myocardial infarction was diagnosed when there was typical cardiac pain at rest with abnormal $Q$ waves in the electrocardiogram or a twofold or greater rise in serially measured cardiac enzymes (serum glutamic oxaloacetic acid transaminase, creatine kinase, and lactate dehydrogenase). Unstable angina was diagnosed when there was cardiac pain at rest with serial ST and T wave changes in the electrocardiogram but with normal or a less than twofold increase of cardiac enzymes.

Patients were classified as non-smokers if they had never regularly smoked as many as one cigarette a day, ex-smokers if they had smoked less than one cigarette a day for at least three months before admission, and current smokers if they had regularly smoked at least one cigarette a day in the three months before admission. Pipe and cigar smoking were noted, as was the age at which ex-smokers had stopped. Current and former pipe or cigar smokers were classified as current or ex-smokers in the same way as cigarette smokers. Patients had to have stopped for three months to be classified as an exsmoker. Total serum cholesterol and high density lipoprotein fraction were assayed in blood samples taken, in most cases, within 12 hours of onset of acute symptoms.

Patients were categorised as hypertensive $(a)$ if the mean of all blood pressure measurements taken on the fourth day after admission was $\geqslant 90 \mathrm{~mm} \mathrm{Hg}$; (b) if the patient was known to have been treated for hypertension before admission; (c) if at least two of the following three criteria were present:

(1) past history of hypertension detected by a physician (whether or not it was treated),

(2) electrocardiographic evidence of left ventricular hypertrophy,

(3) hypertensive retinopathy.

A multiple logistic regression was calculated to find the best combination of age, cholesterol, and hypertension to discriminate between current smokers and non-smokers. Scores on the function were then computed for ex-smokers as well as for current smokers and non-smokers. Ex-smokers were classified in five year groups, according to the length of time since they had given up. We compared mean function scores between groups by Tukey's procedure to correct for errors arising from repeated comparison of means. ${ }^{10}$

\section{Results}

Nine hundred and eighty seven patients were admitted to the study. Ten of these had to be excluded from the analysis because we could not record their smoking habits. There were 639 men $(65.4 \%)$ and 338 women (34.6\%). Two hundred and four patients $(20.9 \%)$ were admitted with unstable angina and 773 $(79.1 \%$ ) with myocardial infarction, of whom $49.7 \%$ had a complicated in-hospital course. There were 515 current smokers, 264 ex-smokers, and 198 nonsmokers. Because many data were missing we examined the relation between missing data and smoking state (table 1). Hypertension could not be assessed in 28 cases, age had not been recorded in 7 , and serum cholesterol had not been measured in 201 .

Table 2 shows the relation between lifetime smoking habits and risk factors. When patients were examined in three groups-non-smokers, ex-smokers, and current smokers-there were significant differences in the distributions of age, cholesterol, and hypertension. The significance values for these differences are shown below table 2 . When individual groups were compared, all three groups differed significantly $(p<0.05)$ from one another in age and the proportion with hypertension. The mean serum concentration of cholesterol was significantly different in non-smokers $(6.51 \mathrm{mmol} / \mathrm{l})$ and ex-smokers $(6.10 \mathrm{mmol} / \mathrm{l})$, but the concentration in current smokers $(6.25 \mathrm{mmol} / \mathrm{l})$ did not differ significantly from either.

A direct logistic regression analysis performed on current and non-smokers yielded significant independent predictive associations between smoking and both age and hypertension (both $p<$ 0.0001 ). Although cholesterol was not significantly independently associated with smoking after allowance for age and hypertension $(p=0.068)$, it was retained in the model in view of its borderline probability. The resulting function, then, is a combination of age, cholesterol, and hypertension calculated so as to place smokers at one end of a range of risk and non-smokers at the other. We refer to it, for simplicity, as a discrimination score. To examine the

Table 1 Distribution of missing data in the study group (number of valid cases, followed by number of missing cases in parentheses)

\begin{tabular}{lcrcc}
\hline Smoking state & Cholesterol & Age & $\begin{array}{l}\text { Hyper- } \\
\text { tension }\end{array}$ & $\begin{array}{l}\text { All three } \\
\text { variables }\end{array}$ \\
\hline Current smokers & $431(84)$ & $512(3)$ & $501(14)$ & $424(91)$ \\
Ex-smokers & $197(67)$ & $264(0)$ & $257(7)$ & $196(68)$ \\
For $<5$ yr & $67(26)$ & $93(0)$ & $91(2)$ & $67(26)$ \\
For 6-10 yr & $42(15)$ & $57(0)$ & $56(1)$ & $41(16)$ \\
For 11-15 yr & $28(7)$ & $35(0)$ & $34(1)$ & $28(7)$ \\
For 16-20 yr & $34(8)$ & $42(0)$ & $42(0)$ & $34(8)$ \\
For $>20$ yr & $26(11)$ & $37(0)$ & $34(3)$ & $26(11)$ \\
Never smoked & $148(50)$ & $194(4)$ & $197(1)$ & $145(53)$ \\
\hline
\end{tabular}

Data on length of time since the patient stopped smoking were missing in 10 of 264 cases. 
Table 2 Risk factors in current smokers, non-smokers, and ex-smokers

\begin{tabular}{|c|c|c|c|c|}
\hline Category & $\begin{array}{l}\text { Cholesterol } \\
\text { (mean }(S E))(\operatorname{mmol} / l)\end{array}$ & $\begin{array}{l}\text { Percentage } \\
\text { hypertensive (SE) }\end{array}$ & Age (yr) (mean (SE)) & $\begin{array}{l}\text { Discrimination } \\
\text { score }(S E)\end{array}$ \\
\hline $\begin{array}{l}\text { Current smokers } \\
\text { Ex-smokers } \\
\text { For }<5 \text { yr } \\
\text { For } 6-10 \text { yr } \\
\text { For } 11-15 \text { yr } \\
\text { For } 16-20 \text { yr } \\
\text { For }>20 \text { yr }\end{array}$ & $\begin{array}{l}6.3(0.07) \\
6.1(0.09) \\
6.1(0.22 \\
6.0(0.16) \\
6.0(0.23) \\
6.2(0.24) \\
6.1(0.26)\end{array}$ & $\begin{array}{l}25 \%(2 \cdot 0) \\
36 \%(3 \cdot 2) \\
38 \%(6 \cdot 4) \\
27 \%(6 \cdot 4) \\
35 \%(8 \cdot 6) \\
43 \%(8 \cdot 0) \\
38 \%(8 \cdot 7)\end{array}$ & $\begin{array}{l}59(0.5) \\
63(0.7) \\
60(1.5) \\
61(1.5) \\
60(1.6) \\
64(1.6) \\
69(1 \cdot 2)\end{array}$ & $\begin{array}{l}1.00(0.009) \\
0.91(0.015) \\
0.90(0.028) \\
0.98(0.030) \\
0.96(0.042) \\
0.89(0.037) \\
0.81(0.033)\end{array}$ \\
\hline Never smoked & $\begin{array}{l}6.5(0.13) \\
F=3.56 \\
p=0.029\end{array}$ & $\begin{array}{l}51 \%(3.6) \\
\chi^{2}=49.38 \\
p<0.001\end{array}$ & $\begin{array}{l}66(0.8) \\
F=47.98 \\
p<0.001\end{array}$ & $\begin{array}{l}0.80(0.020) \\
F=68.4 \\
p<0.001\end{array}$ \\
\hline
\end{tabular}

^Based on age, cholesterol, and hypertension (see text).

Cholesterol: $1 \mathrm{mmol} / 1=38.6 \mathrm{mg} / \mathrm{dl}$.

Statistics are for three way classification of smokers (non, ex, and current).

For comparison of pairs of means, see text.

position of ex-smokers on this range we calculated their scores according to this function. Table 2 shows the mean function score for current smokers, exsmokers, and non-smokers. All three groups differed significantly from one another at an alpha level of 0.05 .

In order to determine how long after they stop smoking ex-smokers continue to have a significantly higher risk than non-smokers, we divided ex-smokers into groups based on the interval since they stopped, divided up into five year periods. Table 2 shows that the risk factor score based on the logistic regression declined with length of time since smoking was stopped. However, only those patients who had given up at least 15 years before their first episode of acute coronary heart disease had a mean risk score that did not differ significantly $(p>0.05)$ from the mean risk score of a non-smoker.

\section{Discussion}

Smoking is a major risk factor for acute coronary heart disease. Furthermore, it is widely accepted that those who stop smoking reduce their risk. The most optimistic view is that risk declines rapidly after the end of smoking and soon resembles that of nonsmokers. ${ }^{28}$ This implies that the role of smoking as a risk factor in coronary heart disease is not primarily atherogenic, a view which has received some support from angiographic investigations. ${ }^{11} \mathrm{~A}$ recent report from the British Regional Heart Study, on the other hand, found no evidence of a rapid decline in risk, and other studies have shown a continuing excess risk lasting for up to twenty years. ${ }^{34}$

Several of the studies showing no lasting effect of smoking on risk of acute coronary heart disease were hampered by small event rates. ${ }^{68}$ Furthermore, the endpoints studied ranged from fatal coronary disease through non-fatal infarction to any manifestation of acute coronary ischaemia, as in the case of the present study. So, despite a considerable body of research the two central questions remain unanswered: is there a drop in risk immediately after the end of smoking and for how long does a residual additional risk remain? We attempted to cast light on these questions by making use of hospital data. The technique used does not allow an estimate of the absolute level of risk because there is no denominator population and inevitably the data are incomplete. Nevertheless, it is based on a large series of coronary events which were scarce in prospective studies of populations.

The method we used assumed that non-smokers who developed coronary heart disease did so because of higher levels of other risk factors-age, hypertension, and/or cholesterol. Accordingly, if an acute episode of coronary heart disease develops in exsmokers with a lower loading of other risk factors than non-smokers, the ex-smokers must have a residual risk associated with smoking which acts on their advanced coronary artery disease to precipitate a first acute coronary event.

Ex-smokers who had given up recently tended to be younger than ex-smokers who had given up for longer. One explanation for this is that older exsmokers were more likely to have given up for longer simply because they were older. Two factors, however, argue against this interpretation: the first is that the increase in age from current smokers through ex-smokers to non-smokers is consistent with the hypothesis that the lower the person's exposure to smoking, the slower the progression of atherosclerosis. Secondly, our findings accord with those of Cook et al who reported no immediate decline in cardiovascular risk when smoking stopped, and a continuing risk persisting for up to 20 years. ${ }^{1}$ Further analysis showed a dose response relation between the number of years of smoking and subsequent risk. Since we had no information on the age at which our patients started smoking we could not analyse our data in this way. A recent report on smoking, clotting 
factors, and coronary heart disease risk by the Northwick Park Heart Study found that it took five years of non-smoking for clotting factors in exsmokers to fall to values in non-smokers. ${ }^{12}$ In exsmokers there was a relation between total years smoking and concentrations of clotting factor. These findings may in part explain both the results presented here and those of the British Regional Heart Study. ${ }^{1}$ We can offer no explanation for the difference between the effect of stopping smoking on coronary risk in the general population, which seems to be gradual and small, and the immediate halving of the risk that we reported in patients who gave up smoking after an episode of acute coronary heart disease. ${ }^{13}$ Such patients seem to be more susceptible to the effects of smoking than the general population, but no mechanism for this increased susceptibility has yet been established.

Serum concentrations of cholesterol were lowest in ex-smokers in our patient group, intermediate in current smokers, and highest in non-smokers. It is likely that ex-smokers make other lifestyle changes aimed at risk reduction and these reduce their cholesterol concentrations to below values in their smoking counterparts. The role of such lifestyle changes as a possible confounding factor in the association between giving up smoking and subsequent coronary risk should be studied.

The mode of action of smoking in the causation of coronary heart disease is still unproven. It is likely, indeed, that it acts in two ways. First, smoking may increase the rate of atherosclerosis for any given serum concentration of cholesterol, thus bringing forward the age at which atherosclerosis will be sufficiently advanced to lead to an acute ischaemic event. Secondly, smoking may exert vasospastic and thrombogenic effects, thus precipitating an acute coronary event when the degree of atherosclerosis is less than would be required for a coronary event in a non-smoker. The rapid decline in risk found by some studies may reflect the removal of the effect of smoking in precipitating an infarction, while the continuing risk reported by others presumably reflects a continuing atherogenic effect of previous smoking. Our data provide support for this latter effect, indicating that although there is a suggestion of a lowering of risk immediately after the end of smoking, the risk factor levels found in ex-smokers are not distinguishable from those in current smokers until 15 years after they stop smoking; this suggests a continuing smoking associated risk in this period. So, although it is never too late to give up smoking nor is it ever too early.

We thank the medical records department of St Vincent's Hospital for their considerable help in the collection of the data presented.

\section{References}

1 Cook DG, Shaper AG, Pocock SJ, Kussick SJ. Giving up smoking and the risk of heart attacks: a report from the British Regional Heart Study. Lancet 1986;ii: 1376-80.

2 Royal College of Physicians Report. Smoking or health. London: Pitman, 1977.

3 Jenkins CD, Rosenman RH, Zyzanski SJ. Cigarette smoking: its relationship to coronary heart disease and related risk factors in the Western Collaborative Study. Circulation 1968;38:1140-55.

4 Doll R, Gray R, Hafner B, Peto R. Mortality in relation to smoking: 22 years observation on female British doctors. Br Med J 1980;i:967-71.

5 Royal College of Physicians report. Health or smoking. London: Pitman, 1983.

6 Rosenberg L, Kaufman D, Helmrich S, Shapiro S. The risk of myocardial infarction after quitting smoking in men under 55 years of age. $N$ Engl J Med 1985; 313:1511-4.

7 Gordon T, Kannell WB, McGee D. Death and coronary attacks in men after giving up smoking: a report from the Framingham Study. Lancet 1974;ii:1345-8.

8 Jajich CL, Ostfeld AM, Freeman DH Jr. Smoking and coronary heart disease mortality in the elderly. JAMA 1984;252:2831-4.

9 Conroy RM, Mulcahy R, Hickey N, Daly L. Is a family history of coronary heart disease an independent coronary risk factor? Br Heart $J$ 1985;53:378-81.

10 SAS Institute Inc. SAS user's guide: statistics, Version 5 Edition. Cary, NC: SAS Institute 1985:470-6.

11 Hartz AJ, Barboriak PN, Anderson AJ, Hoffmann RG, Barboriak JJ. Smoking, coronary artery occlusion and nonfatal myocardial infarction. JAMA 1981;246: 851-3.

12 Meade TW, Imeson J, Stirling Y. Effects of changes in smoking and other characteristics on clotting factors and the risk of ischaemic heart disease. Lancet 1987;ii:986-8.

13 Mulcahy R. Influence of cigarette smoking on morbidity and mortality after myocardial infarction. $\mathrm{Br}$ Heart $J$ 1983;49:410-5. 